\title{
La tortue marine au Cameroun, genre Lepidochelys : nidification, biometrie de Lepidochelys olivacea (Eschscholtz, 1829) (Reptilia, Cheloniidae) dans la réserve de faune de Campo (Sud Cameroun)
}

\author{
Hyacinthe ANGONI ${ }^{1 *}$, AMOUGOU AKOA ${ }^{1}$, C.F. BILONG BILONG ${ }^{2}$ et \\ Jacques FRETEY ${ }^{3}$
}

\begin{abstract}
${ }^{1}$ Université de Yaoundé I, Département de Biologie et Physiologie Végétales Faculté des Sciences BP. 812
${ }^{2}$ Université de Yaoundé I, Département de Biologie et Physiologie Animale. Faculté des Sciences BP. 812

${ }^{3}$ Fédération française des Sociétés de Sciences Naturelle, Muséum National d'Histoire Naturelle, 43-57, rue Cuvier, Paris, France Coordonnateur du Programme KUDU.

*Auteur correspondant, E-mail : angonih@yahoo.fr
\end{abstract}

\section{RESUME}

Ce document présente les données recueillies sur Lepidochelys olivacea le long des côtes de la réserve de Campo-Ma'an au Cameroun. Les études menées de 1998 à 2005 ont consisté en un recensement des espèces venant pondre sur les plages et une délimitation des périodes de pontes. Des mesures ont été également effectuées sur les spécimens rencontrés et à partir des carapaces collectées. Les résultats montrent que, Lepidochelys olivacea pond sur les côtes de l'UTO Campo Ma'an entre octobre et mars, avec un taux élevé de ponte entre novembre et janvier. La longueur moyenne $(68,98 \mathrm{~cm})$ et la largeur moyenne $(68,66 \mathrm{~cm}) \mathrm{de}$ la courbe de la dossière ont été observées sur les sites pour 118 Lepidochelys olivacea marquées sur les plages du Cameroun. L'identification des carapaces a montré la présence des juvéniles (19-20), subadultes (40-45) et les adultes de Lepidochelys olivacea au Cameroun.

(C) 2010 International Formulae Group. All rights reserved.

Mots clés : Lepidochelys olivacea, plage de ponte, nidification, saison de ponte.

\section{INTRODUCTION}

Les plages et les berges d'une mer ou d'un océan sont à la fois les lieux d'une activité humaine intense, le milieu de vie de nombreuses espèces animales et végétales, et enfin le milieu unique de reproduction de certains animaux. La préservation de ces milieux des activités pouvant entraîner leur dégradation et leur valorisation sont vitales pour éviter un écocide en ce début du XXI ${ }^{\text {ème }}$ siècle. Quatre espèces de tortues fréquentent les eaux marines camerounaises. Il s'agit de la tortue luth (Dremochelys coriacea), la tortue olivâtre (Lepidochelys olivacea), la tortue verte (Chelonia mydas), la tortue imbriquée (Eretmochelys imbricata).

La tortue Chelonia mydas et Eretmochelys imbricata, vivent autour des rochers colonisés par des algues et des gorgones tandis que la Dermochelys coricea et Lepidochelys olivacea pondent sur les plages de sable.

Les tortues qui pondent sur les côtes de la réserve de Campo-Ma'an sont 
confrontées aux problèmes engendrés par la présence d'un milieu marin où règne une activité de pêche intense entraînant les prises accidentelles des espèces menacées et une urbanisation non contrôlée des milieux côtiers (Fretey, 1998). Pourtant la reproduction et la survie des jeunes Lepidochelys olivacea vont par conséquent dépendre de ces deux habitats.

La fréquence des tortues olivâtres Lepidochelys olivacea venant pondre sur les plages est de plus en plus irrégulière sur les côtes de la Réserve de Campo-Ma'an, ses individus font l'objet d'une exploitation par les populations qui consomment leur viande, leurs œufs et pratiquent le commerce des carapaces.

Les tortues sont inscrites dans la liste rouge des espèces en menaces critiques (Seminof et Shanker, 2008), mais sur le plan national la tortue olivâtre ne bénéficie que d'une protection partielle dont, classées dans la classe $B$ (espèces à abattage réglementé) (Annonyme, 1954).

La tortue olivâtre est très fréquente sur les côtes Sud du Cameroun, très bien connu par les populations iyassa qui l'ont nommé «kudu amoundja » car elle dépose ses œufs sur les plages chaque année, elle est capturée sur les filets de pêche et sa carapace est vendue aux touristes. Mais les périodes de nidification et la taille de ces espèces sur les côtes Sud du Cameroun restent mal connues.

Pour contribuer à la connaissance des aspects biologiques de ces espèces au Cameroun, nous nous proposons de:

- identifier les sites et les périodes de ponte ;

- étudier les caractéristiques biométriques des espèces rencontrées sur les plages de pontes ;

- identifier les classes de taille de Lepidochelys olivacea capturée au filet de pêche.

\section{MATERIEL ET METHODES \\ Site d'étude}

L'Unité Technique Opérationnelle (UTO) de Campo Ma'an, créée le 06 août 1999 par arrêté Nº1999/054/PM, d'une superficie de 709760 ha, s'étend sur trois départements de la Région du Sud, dont le département de l'Océan bordé par les côtes sableuses. Cette UTO comprend une zone essentielle de protection intégrale (parc national) et une zone périphérique d'utilisations multiples (zone tampon) (De Cam et al., 2002).

Pour cette étude, cinq sites ont été retenus. Il s'agit du Sud vers le Nord des localités de Mbendji, Ebodjé, Ipeyendjé, Beyo, Lolabé ayant respectivement, 2 ; 3 ; 2,5 ; 2,7 et 2,8 km de côtes marquées par l'existence d'un écran végétal naturel permettant la stabilité de la ligne côtière (Figure 1).

\section{Méthodes}

L'étude s'est déroulée pendant une période de six années et elle a été subdivisée en deux étapes dont un inventaire des tortues marines et une identification des carapaces dans les villages côtiers. L'objectif principal de l'étude était de déterminer les périodes de nidification et contribuer à la connaissance de quelques éléments morphologiques de Lepidochelys olivacea sur les côtes de l'UTO Campo Ma'an.

\section{Etude des périodes de ponte}

Les pontes de tortues marines chevauchent entre deux années (la fin de l'année précédente et le début de l'année suivante appelé saison de ponte). Ainsi trois saisons de ponte (1998/1999, 2000/2001, 2004/2005), ont permis de récolter les données pour la détermination des périodes de ponte de Lepidochelys olivacea et à l'aide de la méthode de Richardson (1999) et celle préconisée par l'ONG (PROTOMAC) Protection des Tortues Marines en Afrique Centrale (Fretey, 1998) sur les plages situées entre Mbendji et Bekolobe. 


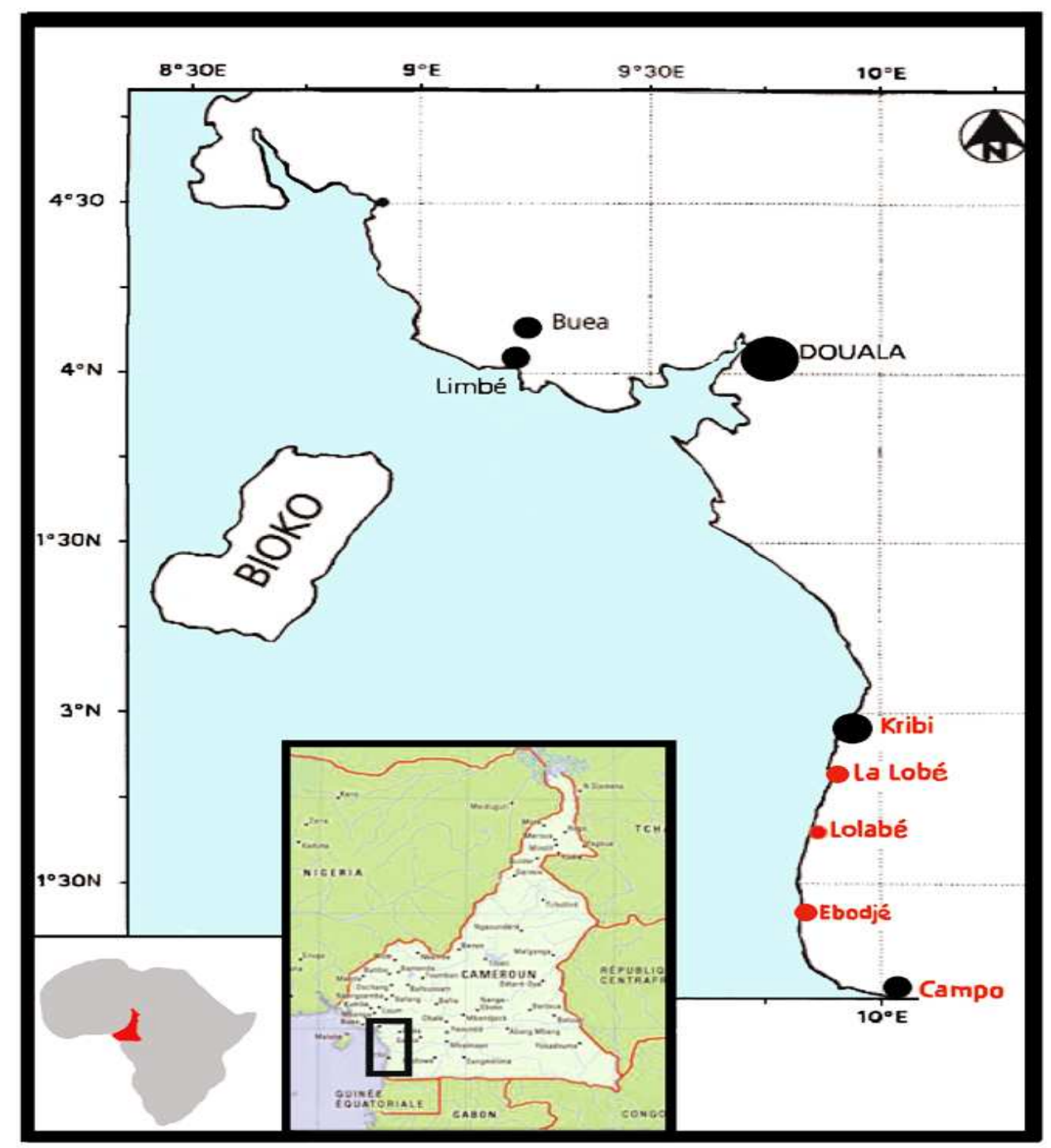

Figure 1: Zone côtière de la réserve de Campo Ma'an.

Quotidiennement, deux patrouilles nocturnes ont été effectuées en marée basse, dans chacun des cinq sites de plage entre 20 heures et 2 heures pour identifier directement les tortues venues pondre sur les plages. Les femelles venues pondre sur la plage ont directement été identifiées et mesurées (longueur et largeur de la courbe de la dossière et la largeur maximale de la tête des animaux) A l'aube entre 4 heures et 5 heures une seconde patrouille a été effectuée pour identifier les traces et localiser les nids de tortues marines.

\section{Etude de la biométrie des tortues marines}

Pendant six années (1998, 1999, 2000, 2001, 2004 et 2005), grâce aux carapaces exposées dans les ménages et vendues le long de l'axe routier Kribi-Campo, l'étude morphologique des tortues olivâtres Lepidochelys olivacea rencontrées sur les plages camerounaises a été complétée. 


\section{RESULTATS}

Les pontes des tortues Lepidochelys olivacea sur les plages de la Réserve de Campo-Ma'an sont fréquentes entre les sites de Mbendji et de Bekolobé long de 13 kilomètres et suivi quotidiennement.

\section{Biologie de ponte de Lepidochelys olivacea Suivi des plages de ponte}

Les observations faites pendant les patrouilles sur les plages entre juillet 2000 et août 2001 montrent que, entre Mbendji et Bekolobe, il y a des pontes des tortues marines d'espèce Lepidochelys olivacea. Toutes les plages n'enregistrent pas le même nombre de montées sur les plages pour pondre des œufs. Toutefois, les plages de la zone de suivi d'une longueur de 13 kilomètres enregistrent les montées régulières en période de nidification. Cette zone située entre Mbendji et Bekolobé (Lolabé) est favorable à la nidification avec 143 nids, 51 en $1998 / 1999$, pendant la saison 2000/2001, 86 en 2004/2005 de Lepidochelys olivacea répartis le long des 13 kilomètres de longueur de site d'étude.

Le Tableau 1 présente les sections de la plage leurs longueurs et le nombre de tortues recensées pendant les saisons 1998/1999, 2000/2001, 2004/2005.

Le site d'Ipeyendjé a enregistré beaucoup plus de montées de tortues pour pondre suivi du site de Mbendji et de Bekolobe à cause de leur relative isolation visà-vis des activités anthropiques. Tandis que qu'Ebodjé enregistre peu de ponte à cause d'une intense activité de la pêche et l'urbanisation poussée des côtes.

\section{Période de ponte}

Des observations faites pendant trois saisons années (1998/1999, 2000/2001, 2004/2005), ont permis de circonscrire une période de ponte qui débute en octobre et se termine en mars. Le mois qui enregistre le plus nombre de ponte de tortues Lepidochelys olivacea (Figure 2) sur les plages étudiées est le mois de décembre pour toutes les trois saisons d'étude.
Les pontes chevauchent entre deux années (octobre de l'année précédente à mars de l'année suivante). Cette période de l'année est appelée, une saison de ponte. On observe cependant quelques pontes sporadiques en août, septembre et avril. En fonction des mois, le nombre de nids par saison de ponte varie d'une année à l'autre. La Figure 3 ci-dessous présente la distribution des pontes en fonction des mois (saisons de ponte 98/99, 2000/2001, 2004/2005).

\section{Biométrie \\ Taille des individus}

Sur les plages de ponte, des mesures biométriques effectuées sur 118 tortues Lepidochelys olivacea montrent que, dans l'ensemble, les valeurs moyennes en taille des tortues Lepidochelys olivacea sont de 68,98 de longueur $(87-56,5 \mathrm{~cm})$ et $68,66(82-60,5 \mathrm{~cm})$.

L'analyse combinée des tortues capturées accidentellement baguées et les tortues tuées dont les carapaces étaient en vente aux abords des routes a permis de déterminer la structure en taille de cette espèce au Cameroun.

Ainsi, la structure en taille (longueur et largeur de courbe de la dossière) de Lepidochelys olivacea en fonction des classes de longueurs montre l'existence (Figure 4) de trois groupes :

- les juvéniles qui regroupent les animaux de 15 à $20 \mathrm{~cm}$ de longueur;

- les subadultes dont la longueur de la courbe de la dossière des animaux varie de 40 à $55 \mathrm{~cm}$;

- les adultes qui regroupent les animaux dont la longueur est supérieure ou égale à $60 \mathrm{~cm}$.

Les deux premiers groupes sont très faiblement représentés dans les échantillons identifiés. C'est dans le dernier groupe que se situent de nombreux animaux entre 60 et 95 $\mathrm{cm}$ Ce qui se justifie par le fait que l'espèce fréquente les côtes camerounaises pendant la période de nidification. 


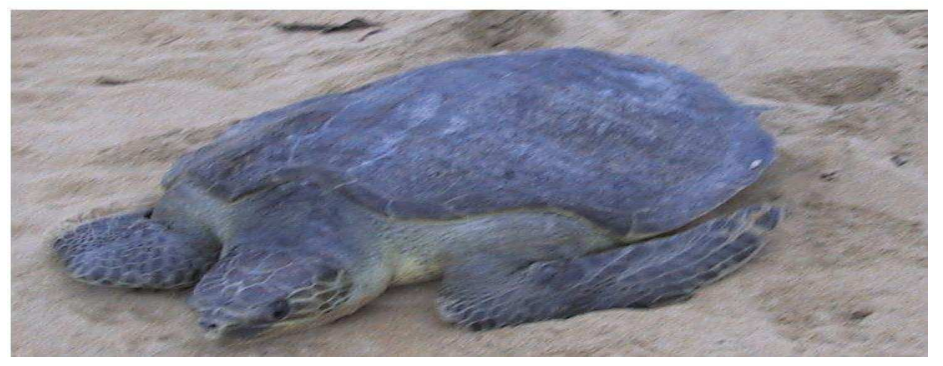

Figure 2 : Tortue Lepidochelys olivacea.

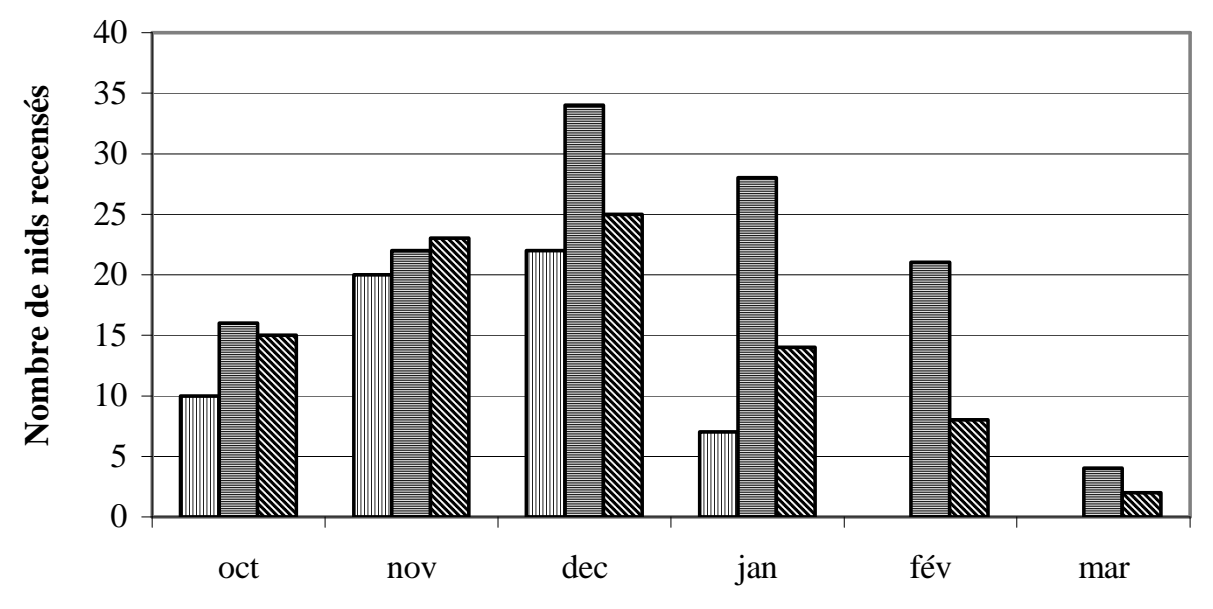

Mois de l'année

Figure 3 : Distribution des pontes en fonction des mois (saisons de ponte 1998/1999, 2000/2001, 2004/2005).

IIIIII Saison de ponte 1998/1999 Saison de ponte 2000/2001 WW Saison de ponte 2004/2005

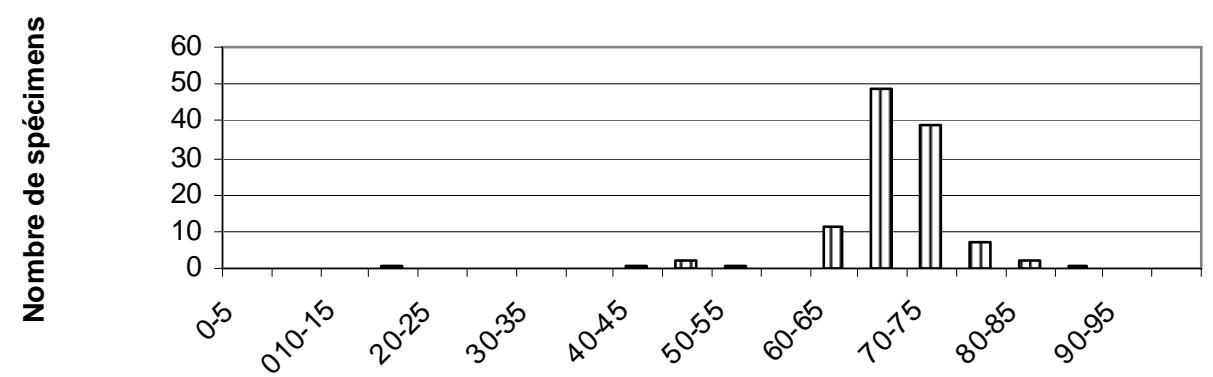

classes de longueurs en $\mathrm{cm}$

Figure 4 : Répartition des spécimens de Lepidochelys olivacea capturés sur les côtes en fonction des classes de la longueur de la courbe de la dossière. 
Tableau 1 : Nombre de nids par localité au cours des saisons 1998/1999, 2000/2001, 2004/2005.

\begin{tabular}{lllll}
\hline $\begin{array}{l}\text { Plage de ponte } \\
\text { Lepidochelys olivacea }\end{array}$ & de $\begin{array}{l}\text { Longueur de la } \\
\text { plage }(\mathbf{k m})\end{array}$ & \multicolumn{3}{c}{$\begin{array}{c}\text { Nombre de nids identifiés par site et par } \\
\text { saison de ponte }\end{array}$} \\
\cline { 3 - 5 } & & $\mathbf{1 9 9 8 / 1 9 9 9}$ & $\mathbf{2 0 0 0 / 2 0 0 1}$ & $\mathbf{2 0 0 4 / 2 0 0 5}$ \\
\hline Mbendji & 2 & 16 & 30 & 17 \\
Ebodjé & 3 & 10 & 06 & 6 \\
Ipeyendjé & 2,5 & 15 & 58 & 37 \\
Beyo & 2,7 & 8 & 26 & 13 \\
Bekolobé & 2,8 & 2 & 23 & 13 \\
\hline
\end{tabular}

\section{DISCUSSION}

La tortue olivâtre (Lepidochelys olivacea) nidifie sporadiquement à partir de septembre sur les côtes de l'UTO CampoMa'an. On a relevé un nombre élevé de nids au mois de décembre et les pontes diminuent en janvier. La fin de la saison de ponte se situe au mois de mars. Les enquêtes réalisées par Fretey (1998) auprès des pêcheurs locaux confirment que la période de ponte débute en octobre et se termine en mars avec un pic élevé de ponte en décembre.

On a noté que la tortue olivâtre (Lepidochelys olivacea) est très fréquente sur les plages de l'UTO Campo-Ma'an pendant les périodes de ponte. Ceci confirme également les observations antérieures rapportées par Fretey (1998).

La fréquentation des plages par des tortues Lepidochelys olivacea varie d'une année à l'autre. Ainsi, au cours de la saison 1998/1999, Fretey (1999) indique que, de Mbendji à Bekolobé, les montées sur les plages par ces chéloniens étaient peu nombreuses.

Sur la façade atlantique africaine, les sites de ponte des chéloniens marins varient en fonction des espèces. Ainsi, les tortues olivâtres (Lepidochelys olivacea) pondent au Cameroun, en Guinée Equatoriale (Mba et al., 1998; Formia, 1999), sur l'Ile de Bioko (Castroviejo et al., 1994), à Sao Tomé, au Gabon et en République Démocratique du Congo (Fretey, 2001).

Les périodes de ponte ne sont pas les mêmes. En outre le pic de ponte se situe en décembre au Cameroun, en janvier à Bioko
(Guinée Equatoriale) et entre septembre et octobre au Gabon (Maloueki, 1996).

De l'analyse biométrique de Lepidochelys olivacea identifié sur les plages, on a observé que la taille moyenne des adultes est de 68,98 de longueur $(87-56,5 \mathrm{~cm})$ et $68,66(82-60,5 \mathrm{~cm})$ de largeur de courbe de la dossière pour 118 animaux identifiés. Cette valeur est inférieure à $70,86 \mathrm{~cm}$ que signale Fretey (1999) pour le Cameroun. La taille moyenne des animaux de la population de la côte camerounaise est voisine de la taille des animaux de Sao Tomé et Principe pour laquelle Fretey et al. (2001) indiquent des valeurs moyennes (et étendues) respectives de $68,93 \mathrm{~cm}(62-86)$ et celle des 500 femelles mesurées au Surinam 68,5 cm (63-75). Mais ces moyennes sont légèrement supérieures à celle des 1563 femelles de la plage d'Escobilla au Mexique pour lesquelles Bjorndal (1995) rapporte les valeurs suivantes : $65,2 \mathrm{~cm}(57-$ $72,5)$. Au seuil de $5 \%$ toutes ces moyennes ne sont pas statistiquement différentes entre le Cameroun, Sao Tomé et Principe, Escobilla (Mexique) et Surinam.

Dans la réserve de Campo-Ma'an, nous avons retrouvé de jeunes Lepidochelys olivacea rapportées par Fretey (1998) comme n'ayant jamais été observées dans l'atlantique ouest africaine.

\section{Conclusion}

Le suivi quotidien des plages de l'UTO Campo-Ma'an pour l'étude de l'écologie de ponte des tortues olivâtres Lepidochelys olivacea sur les côtes de l'UTO Campo-Ma'an ont permis de retenir que, la 
tortue olivâtre (Lepidochelys olivacea) pond sur les plages de l'UTO Campo-Ma'an. La fréquence de cette espèce sur ces côtes est régulière entre Mbendji et Bekolobé. Ces espèces pondent sur les plages entre octobre et mars avec un taux élevé de nidification en décembre. Les immatures observés sont rares. Ce qui témoigne de l'importance régionale de la façade maritime de l'UTO Campo-Ma'an.

Les tailles moyennes de Lepidochelys olivacea reste voisines de celles des autres pays autour du Golfe de guinée et des sites de nidification du Mexique et du Surinam.

Il devient donc urgent d'identifier les menaces qui pèsent sur l'espèce et de prévenir sa disparition sur les côtes de la réserve de Campo-Ma'an par des actions concrètes à identifier.

\section{REMERCIEMENTS}

Nous remercions également les organismes tels que Tropenbos International, PROTOMAC (Protection des Tortues Marines en Afrique Centrale) et le WWF KUDU $\mathrm{ZOMBO}$ pour leur soutien financier.

\section{REFERENCES BIBLIOGRAPHIQUES}

Annonyme. 1954. Loi $\mathrm{n}^{\circ}$ 1954/A/MINTOUR/DFAP/SC fixant la liste des animaux des classes A, B et C.et la répartition des espèces animales dont l'abattage est autorisé en groupe ainsi que les latitudes d'abattage par type de permis de chasse. Ministère du Tourisme.

Annonyme. 1999. Arrêté $\mathrm{N}^{\circ}$ 1999/054/PM du 6 août portant la création de l'UTO Campo-Ma'an. Ministère de l'Environnement et des Forêts.

Annonyme. 2000. Décret $\mathrm{N}^{\circ}$ 2000/004/PM du 6 janvier 2000 portant création du Parc National de Campo-Ma'an. Ministère de l'Environnement et des Forêts.

Atangana Etémé R. 1996. Biogéographie des écosystèmes côtiers et marins. Rapport Plan National de Gestion de l'Environnement. 34 p.

Bjorndal KA. 1995. Growth and age of sea turtle. In Biology and Conservation of Sea Turtles (Revised edn), Bjorndal K. A. (ed) ; p 599-600. Proceeding of the World Conference on Sea Turtle Conservation: Washington D.C., 26-30 Novembre 1979. Smithosian Institution Press. 615 p.

Castroviejo J, Juste J, Pérez del Val J, Castel R, Gil R. 1994. - Density and status of sea turtle species in the Gulf of Guinea islands. Biodiversity and Conservation, 3: 828-836.

Chouldhury BC, Panday B, Tripathy B, Andrews HV. 2003. Sea turtle conservation: Eco (turtle) friendly coastal development. AGOI-UNDP Project manual. Center for Herpetology, India, $52 \mathrm{p}$.

De Cam M, Fines JP, Akogo Mvogo G. 2002. Schéma Directeur pour le développement de l'Unité Technique Opérationnelle de Campo Ma'an, Cameroun. Campo Ma'an série 1. Tropenbos International, Wageningen.

Formia A. 1999. Les tortues marines de la baie de Corisco. Canopée, 14( i-ii).

Fretey J. 1998. Statut des tortues marines en Afrique Centrale-Afrique de l'Ouest. 5Le Cameroun. Rapport UICN. 152 p.

Fretey J. 1999. Suivi et conservation des tortues marines dans la réserve de Campo-Ma'an. Rapport du projet CampoMa'an. 40 p.

Fretey J. 2001. Biogéographie et conservation des tortues marines de la côte atlantique d'Afrique. CMS Technical Series Publication, No 6. 428 p.

Fretey J, Dontaine JF, Billes A. 2001. Tortues marines de la façade atlantique de l'Afrique, genre Lepidochelys. 2. Suivi et conservation de L. olivacea (Eschscholtz, 1829) (Cheloni, Chelonidae) à Sao Tomé et Principe. Bull Soc. Herp. Fr., 98: 4356.

Loveridge A, William EE. 1957. Revision of the African tortoises and turtles of suborder Cryptodira. Bull. Museum, 115(6): 163-557.

Maloueki L. 1996. Etude des tortues marines dans la réserve de faune de Conkouati et ses alentours. Rapport final Projet Conkouati, miméogr., non paginé. 
Mba Mba Aetebe J, Nguema J, Garcia JE. 1998. Etude et la conservation des tortues marines sur le littoral de la partie continentale de la Guinée Equatoriale. Canopée, 12(suppl. iii-iv).

MINEF 1999. Profil côtier du Cameroun. Projet Grand Ecosystème Marin du Golfe de Guinée. Ministère de l'Environnement et des Forêts. 106 p.

Ngandjui G. 2001. Etude de la chasse villageoise en vue de sa gestion durable en collaborartion avec les populations résidentes : cas de l'UTO Campo-Ma'an, sud ouest Cameroun. Rapport du séminaire FAO/UICN/TRAFFIC «Links between Biodiversity conservation, Livelihoods and food security: The sustainability use of wild meat», Yaoundé, Cameroun 17-20 Septembre 2001.9 p.
Seminof JA, Shanker K. 2008. Marines turtles and red listing: a review process, the pitffals and novel assessment approaches. Journal of Experimental Marine Biology and Ecology, 356: 52-68.

Richardson JI. 1999. Priorité en biologie de la reproduction et de la ponte. In Research and Management Techniques of the Conservation of Sea Turtles, Karen L, Eckert, Bjorndal KA (eds).

Tomas J, Fretey J, Raga JA, Castroviejo J. 2001. Tortues marines de la façade atlantique de l'Afrique. Genre Lepidochelys. 1. Quelques données concernant la présence de L. olivacea (Eschscholtz, 1829) dans l'île de Bioko (Guinée Equatoriale). 